\title{
Expression of the Chemokine CXCL12 and its Receptors CXCR4 and CXCR7 in Human and Nonhuman Primate Uterine Leiomyomas
}

\author{
Carmen J Booth ${ }^{1}$ and Graciela Krikun ${ }^{2 *}$ \\ ${ }^{1}$ Department of Comparative Medicine, Yale University School of Medicine, USA \\ ${ }^{2}$ Department of Obstetrics, Gynecology \& Reproductive Sciences, Yale University School of Medicine, USA
}

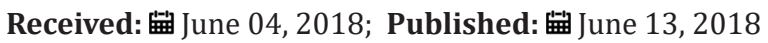

*Corresponding author: Graciela Krikun, Department of Obstetrics, Gynecology \& Reproductive Sciences, Yale University School of Medicine, New Haven, CT, USA

\begin{abstract}
Uterine leiomyomas (also known as fibroids) are associated with several symptoms, including abnormal uterine bleeding, pressure-related symptoms, and recurrent pregnancy loss. These tumors affect up to $80 \%$ of fertile women between the ages of 30 and 50 years. However, there are currently no proven medical therapies for the long-term treatment of leiomyomas, and surgical treatment comes at a significant physical, psychological, and health care costs. It is estimated that this disease results in annual costs of 22 billion dollars. CXCL12 (formerly known as stromal derived factor (SDF-1)) acts via its receptors CXCR4 and CXCR7 and is a potent chemokine found to be excessively expressed in inflammatory diseases. Leiomyomas represent an inflammatory milieu; thus, we posit that the CXCL12/CXCR4/CXCR7 axis is involved in the progression of leiomyomas. This study is a prerequisite for non-hormonal and non-surgical treatment of leiomyomas. When comparing the expression of these molecules in leiomyomas vs. normal myometrium we noted the expression of this axis is elevated in humans and nonhuman primates. Human and nonhuman primates displayed extensive similarities. While CXCR4 stained both nuclei and cytoplasm in leiomyomas, CXCL12 and CXCR7 were confined to the cell membrane and cytoplasm.
\end{abstract}

Keywords: Leiomyomas; Human and nonhuman primates; CXCL12/CXCR4/CXCR7 axis

\section{Introduction}

Leiomyomas are a common benign tumor affecting up to $80 \%$ of women between 30 and 50 years of age [1,2]. These tumors can cause a variety of symptoms, including abnormal uterine bleeding, pelvic pain, bladder or bowel dysfunction, infertility, and recurrent pregnancy loss, and are responsible for more than 200,000 hysterectomies in the United States [1,3-7]. The estimated costs for treatment in the US population exceed $\$ 22$ billion [3]. In addition to pain and infertility, leiomyomas have a significant negative social, psychological impact on the quality of life as reported by $63 \%$ of women with leiomyomas [8]. Current therapies for leiomyomas are not consistently successful. Oral contraceptives are typically the first treatment used for leiomyomas, however treatment failure is common. Progesterone resistance frequently prevents a sufficient clinical response and necessitates other therapies [916]. A gonadotropin releasing hormone ( $\mathrm{GnRH}$ ) agonist is the only drug currently approved by the FDA for treatment of leiomyomas; however, its duration is limited to six month time-course. Patients frequently avoid this drug due to both the high cost and frequent incidence of menopausal side effects [17]. Therefore, there is a major public health need for more effective treatments.

In our current study, we used tissues from humans and nonhuman primates. We demonstrate by immune histochemistry (IHC) that leiomyomas express high levels of the pro-inflammatory chemokine CXCL12 as well as receptor CXCR4. Additionally, we demonstrate high expression of the recently discovered alternative CXCL12 receptor, CXCR7, albeit with different signaling properties [18]. The discovery that CXCR7 is another receptor for CXCL12 with high binding affinity, and recent reports about its involvement in tumor progression, has questioned the potential of "selective blockade" of CXCR4 in malignant tumor therapeutics [19]. The 
over expression of this axis is linked to tumor development, both through a direct modulation of neoplastic cell proliferation, survival, and migration, and, indirectly, by acting on the tumor microenvironment [19]. Leiomyomas are benign tumors and exist in an inflammatory environment. We posit the over expression of CXCL12/CXCR4/CXCR7 axis in leiomyomas make these molecules potential therapeutic targets which may lead to inhibition of disease progression in a non-hormonal fashion. Research studies in several diseases such as inflammatory bowel disease [20], diabetes mellitus [21], and various cancers [18] demonstrate the CXCL12/ CXCR4/CXCR7 protein signaling axis is proinflammatory and furthers disease progression [22]. To date, these pathways have not been elucidated in benign tumors such as leiomyomas.

The etiology of the signaling cascade leading to the development and progression of leiomyomas is unknown. There are no standardized rodent animal models for the study of spontaneous uterine leiomyomas. However, nonhuman primates utilized in breeding colonies and aging studies have shown that spontaneous development of leiomyomas and leiomyosarcomas being the most commonly reported uterine tumors in rhesus macaques [23-26]. Here, we demonstrate by IHC that CXCL12 and both its receptors (CXCR4 and CXCR7) are highly expressed within the leiomyomas of human and nonhuman primates vs. low to no staining in their respective control myometrium. We posit the CXCL12/CXCR4/ CXCR7 signaling creates a feed forward loop within the leiomyomas allowing aberrant cells to further proliferate. We propose that blocking this pathway will lead to new nonhormonal therapies and leiomyoma regression.

\section{Materials and Methods}

Control myometrium and leiomyomas were retrieved as follows: Human tissues were obtained from women undergoing hysterectomy who were consented for acquisition of discarded tissue. Rhesus macaque (RM) tissues were obtained from the diagnostic cases of Dr. Booth. RM \#1, age 20, had a single Results leiomyoma in the myometrium at the level of the cervix. RM \#2, age 24 , had two spatially distinct uterine leiomyomas within the myometrium of the uterus. The nonhuman primates were housed, fed, and cared for, in studies approved by the Yale University School of Medicine Institutional Animal Care and Use Committee. Human and nonhuman primate tissues were immersion-fixed in $10 \%$ neutral buffered formalin, trimmed, cassetted, processed, paraffinembedded, and sectioned at 5 microns. The slides were stained with hematoxylin and eosin (HE) by routine methods in the Department of Comparative Medicine, Mouse Research Pathology Unit. IHC on sections of human and nonhuman primate myometrium and leiomyomas was performed by the Department of Pathology to detect the expression of CXCL12, CXCR4, and CXCR7. Slides were deparaffinized and rehydrated with distilled water. Next, they were placed in epitope retrieval buffer at 95 degrees $\mathrm{C}$ for $30 \mathrm{~min}$, cooled, rinsed, and then placed in tris buffered saline (TBS) with tween (TBST). Endogenous peroxidase was blocked using 3\% hydrogen peroxide then rinsed with TBST. The primary antibody was applied for 30 minutes, and then rinsed in TBST. Next a horseradish peroxidase-conjugated secondary anti-rabbit antibody (The Jackson Laboratory, Bar Harbor, ME) was applied for 30 minutes, and then rinsed in TBST. Liquid 3,3'-diaminobenzidine (DAB, Dako, Carpenteria, CA) chromagen was used and the reaction halted at 5 minutes by placing the slides in distilled water. The slides were then counterstained in hematoxylin, dehydrated, cleared and mounted with resinous mounting media by routine methods. The human HE and IHC slides were examined blind to prior diagnosis (CJB).

a. CXCR4: anti-CXCR4, ab124823, rabbit monoclonal, at 1:1000, Abcam, USA.

b. CXCR7: anti-ACKR3 / CXCR7, LS-B1815-50, rabbit polyclonal, at 1:1000, Lifespan Biosciences, USA.

c. CXCL12: anti-SDF1 / CXCL12, LS-B943-50, rabbit polyclonal, at 1:200, Lifespan Biosciences, USA.

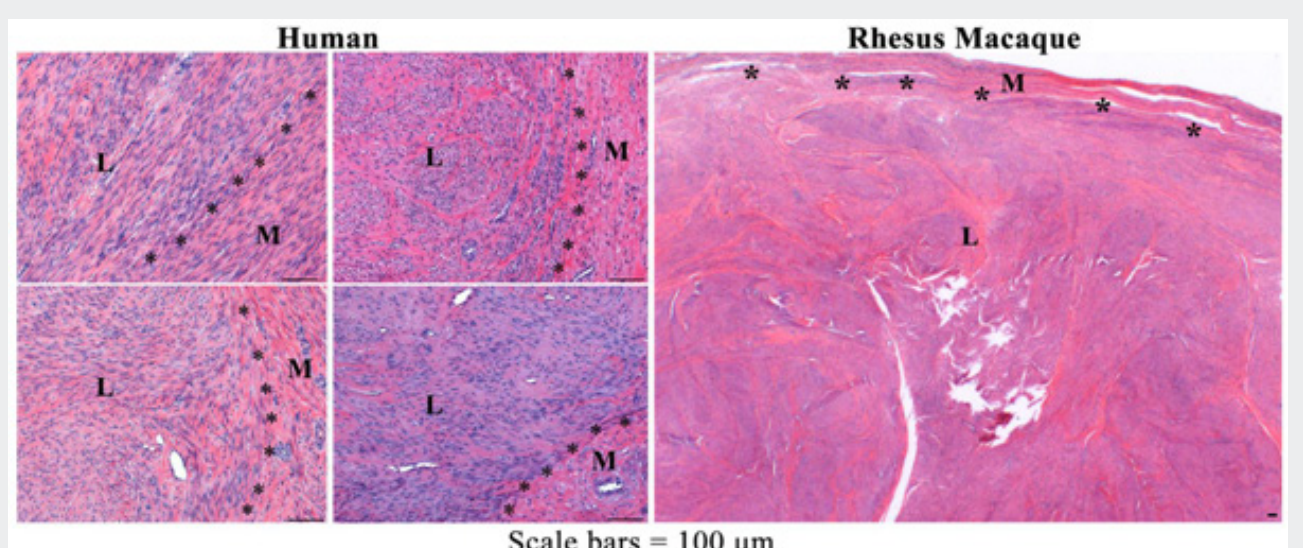

Scale bars $=100 \mu \mathrm{m}$

Figure 1: Representative photomicrographs of HE-stained sections of human (uterine) and rhesus macaque (cervix) leiomyoma (L) and adjacent myometrium (M). 
Uterine leiomyoma in both human and nonhuman primates were characterized as firm, expansile, well-circumscribed, and non encapsulated benign diploid tumors. There may be single or multiple leiomyomas which tend to compress the adjacent myometrium, distort the conture of the uterus, and are microscopically comprised predominantly of smooth muscle with varying amounts of fibrous connective tissue [27-29]. While histologically leiomyomas have low number of mitotic figures they can achieve great size [30]. Representative photomicrographs of HE-stained sections of the human and nonhuman primate leiomyomas used in this study are shown in Figure 1.

\section{CXCR4 Expression in Myometrium vs. Leiomyoma in Humans and Macaques}

While G-protein coupled receptors such as CXCR4 are generally regarded as plasma membrane receptors, it is now believed that in pathological states they can localize to and associate with the nucleus where they retain their function and continue to mediate nuclear signaling [31]. Indeed it has been shown that transportin $\beta 1$-binding to CXCR4 promoted its nuclear translocation [31]. It has been suggested that functional, nuclear CXCR4 may be a mechanism underlying cancer recurrence [18,31,32]. Our preliminary investigation of CXCR4 expression in archival slides of human uterine leiomyomas showed CXCR4 expression in human uteri which consistently stained the majority of leiomyoma tumor cell nuclei $(>50 \%)$ and variable, predominantly weak cytoplasmic staining $(>50 \%)$ in contrast to the adjacent nontumor myometrium which displayed weak to mild staining in markedly fewer adjacent nontumor myometrial smooth muscle cells (nuclei/ cytoplasm<25\%) (Figure 2). Similarly the cervical and uterine nonhuman primate leiomyomas consistently stained strongly positive for the majority of tumor nuclei $(>50 \%)$ and variable, predominantly weak cytoplasmic staining $(<50 \%)$ in contrast to the adjacent nontumor myometrium which displayed weak to mild staining in markedly fewer adjacent nontumor myometrial smooth muscle cells (nuclei/cytoplasm<25\%) (Figure 3).

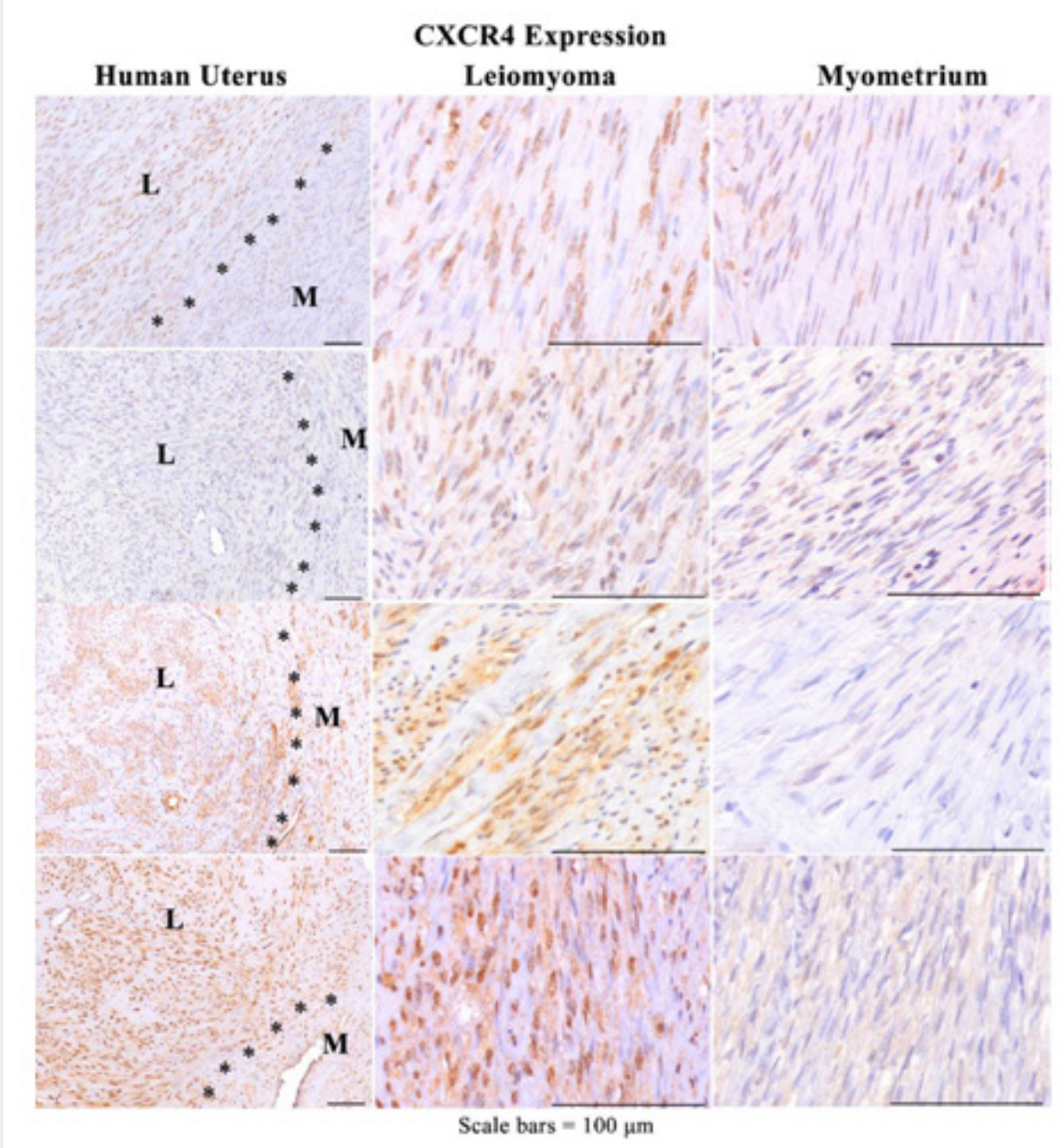

Figure 2: Representative photomicrographs of CXCR4 IHC of human uterine (M) leiomyomas (L) and adjacent myometrium (M) at low and higher power show strong positive staining is primarily nuclear with variable cytoplasmic in tumor cells with fewer, fainter, or no staining in adjacent control myometrium. 


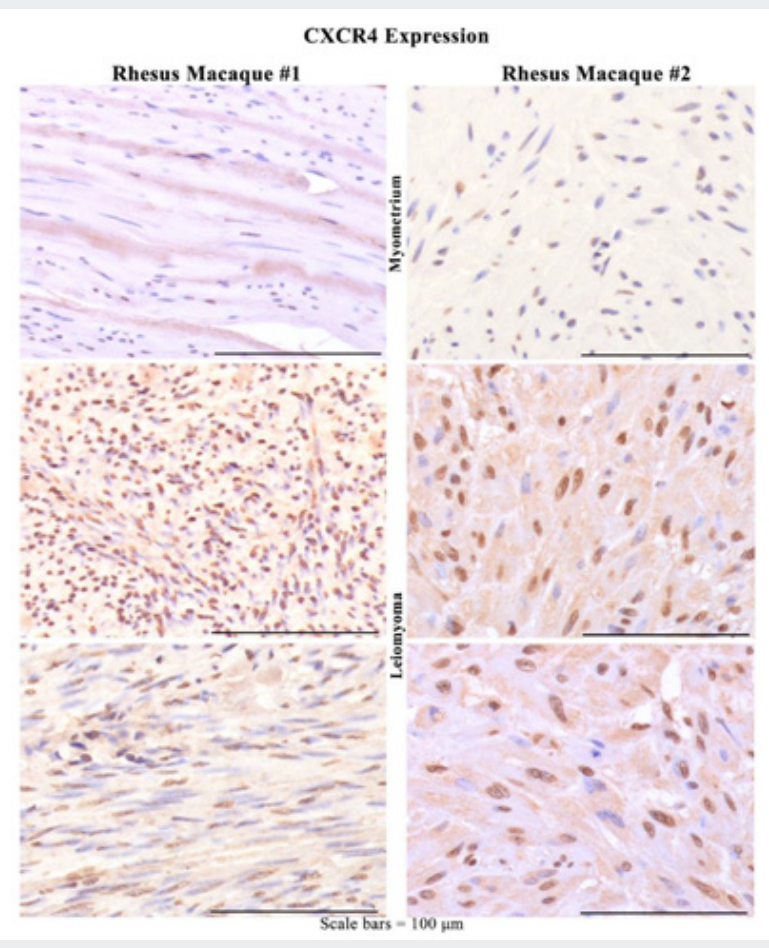

Figure 3: Representative photomicrographs of CXCR4 IHC of rhesus macaque (RM) leiomyomas (L) and adjacent myometrium (M) from the cervix (RM\#1) and two spatially distinct uterine (RM\#2) at high power show strong positive staining is primarily nuclear $(>50 \%)$ with variable cytoplasmic staining in tumor cells with fewer, fainter, or no staining in adjacent control myometrium.

\section{CXCL12 Expression in Myometrium vs. Leiomyoma in Humans and Macaques}

The chemokine CXCL12 possesses an intracellular C-terminal tail which contains serine and threonine residues [33] that can be phosphorylated upon ligand binding eliciting intracellular signaling G protein-recruitment, elevated calcium levels and generation of inositol phosphate [34]. Additionally, $\beta$-arrestin binds to distinct serine residues at the $\mathrm{C}$-terminus determining the $\mathrm{G}$ proteincoupling and initializing the internalization of the receptor (and the bound ligand) which is an important feature in regulation of chemokine function. In recent years, CXCL12 was recognized to participate not only in embryonic development and homeostatic maintenance, but also, in the progression of inflammation [20]. To date, there is a single study investigating the expression of CXCL12 in human leiomyomas vs. control endometrium [35]. The study demonstrated by both RT-PCR and Western blotting, CXCL12 was highly expressed in leiomyomas compared to control myometrium. Interestingly, the expression pattern differed according to the size of leiomyoma [35].

Following our initial studies with CXCR4 (SRI Poster, Abstract F-118:Mar 9, 2018), we next compared CXCL12 expression in human and nonhuman primate leiomyomas by immune histochemistry. As can be seen in Figure 4, both human and macaques demonstrated higher expression of CXCL12 in leiomyomas compared to control myometrium. While staining was clearly expressed in the cytoplasm, CXCL12 was highly expressed in vasculature. This finding is supported by a recent study demonstrating high CXCL12 protein in the endothelium of the pulmonary vasculature [36].

\section{CXCR7 Expression in Human and Macaque Myometrium vs. Leiomyoma}

C-X-C chemokine receptor type 7 (CXCR7) has been reported to regulate cancer cell invasion $[37,38]$. Indeed, recent studies have demonstrated that CXCR4 and CXCR7 are associated with tumor invasion and progression [39]. CXCR7 was identified as an alternate receptor for CXCL12 $[18,20,38]$ which also plays a significant role in inflammatory disease and cancer progression [38]. While studies on the role of CXCR7 before have been conducted in malignant tumors, no such studies have ever been conducted on benign tumors such as leiomyomas. Therefore, we compared the expression of CXCR7 in control myometrium vs. leiomyomas in humans and nonhuman primates. As can be seen in Figure 5, both species demonstrate greater than $75 \%$ of the tumor cells have strong cytoplasmic staining compared to minimal staining in the adjacent myometrium. However, unlike CXCR4, CXCR7 is not expressed in nuclei but largely expressed in the cytoplasm. Moreover, it is very strongly expressed in vessels. It is now known that both CXCR7 and CXCR4 are essential for the tube formation of endothelial progenitor cells in response to CXCL12, indicating that CXCR7 may be another potential target molecule for angiogenesis-dependent diseases [40]. Moreover, the associated expression of CXCR4 and CXCR7 receptors predicts poor prognosis in tumor progression [41]. 


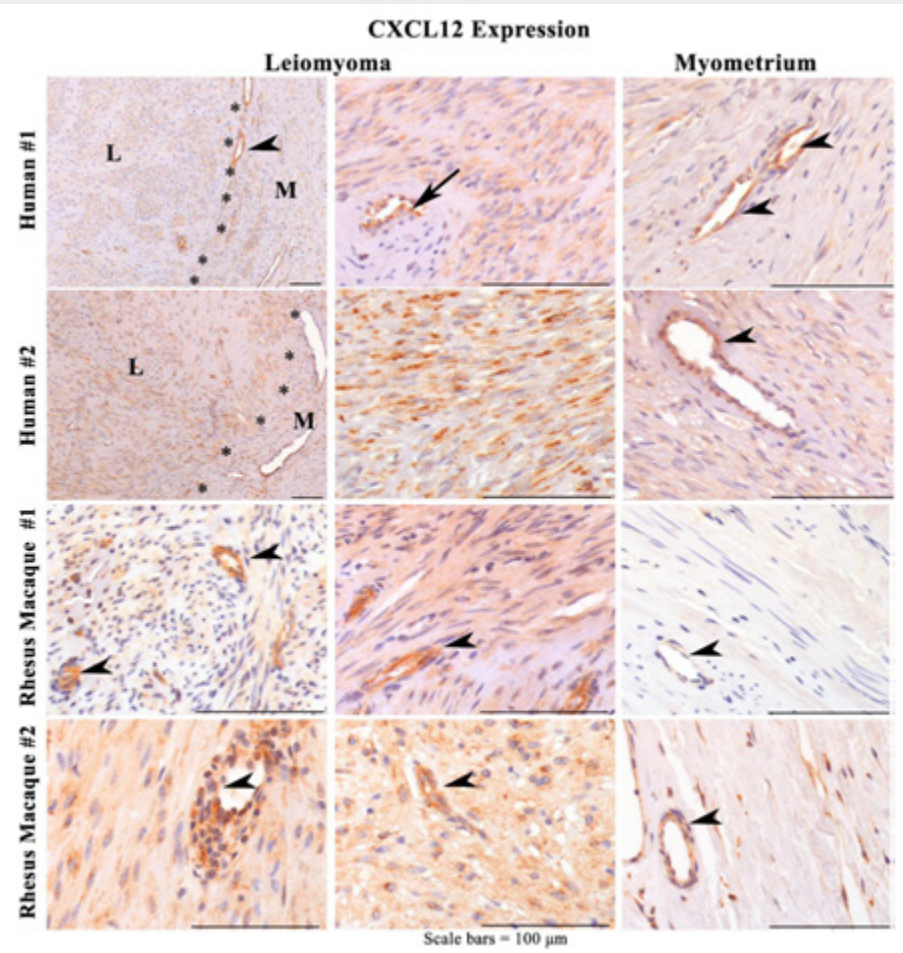

Figure 4: Representative photomicrographs of CXCL12 IHC of human uterine and rhesus macaque cervical (RM\#1) and uterine (RM\#2) leiomyomas (L) and adjacent myometrium (M) at high power show strong positive granular cytoplasmic $(>50 \%)$ in tumor cells with strong vascular endothelial cell staining (arrowhead) and negative vascular smooth muscle cells (arrow) in tumor and adjacent myometrium. There are fewer and fainter, or no staining in cells of adjacent control myometrial smooth muscle cells.

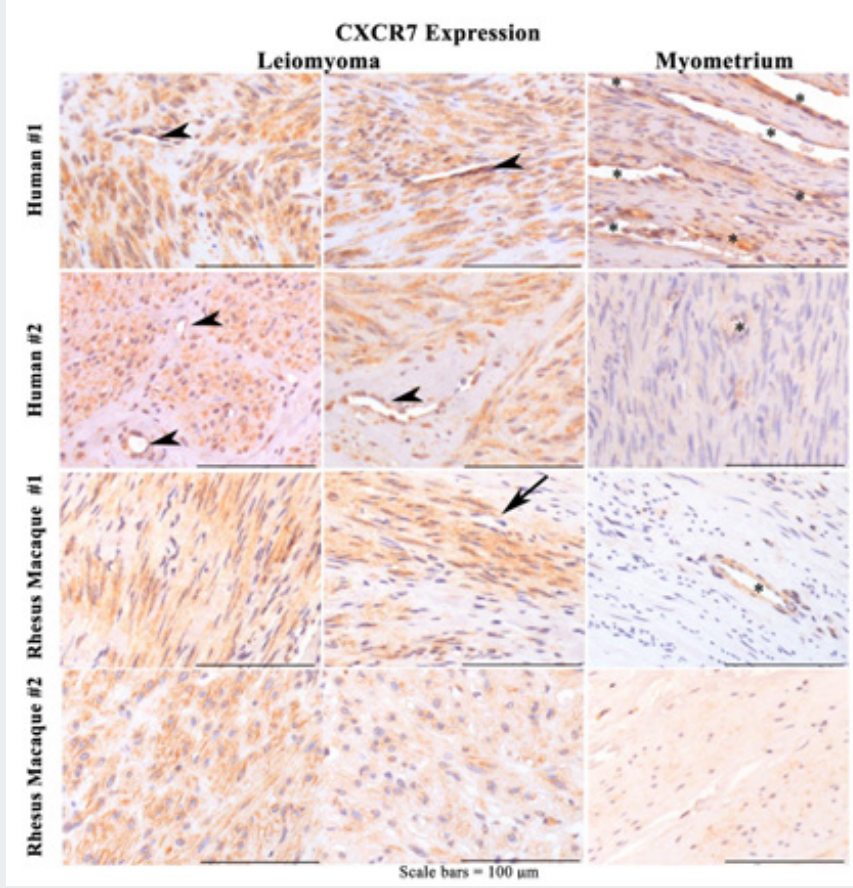

Figure 5: Representative photomicrographs of CXCR7 IHC of human uterine and rhesus macaque cervical (RM\#1) and uterine (RM\#2) leiomyomas (L) and adjacent myometrium $(\mathrm{M})$ at high power show strong positive granular cytoplasmic (>75\%) in tumor cells with variable strong vascular endothelial cell staining (arrowhead) in tumor and adjacent myometrium $\left({ }^{*}\right)$. Scattered vascular endothelial cells are negative (arrow). There are fewer and fainter, or no staining in adjacent control myometrial smooth muscle cells. 


\section{Discussion}

Under physiological conditions, CXCL12 modulates cell proliferation, survival, angiogenesis, and migration initially believed to signal via its traditional receptor CXCR4 $[42,43]$. The function of CXCR7 is generally considered to be mediated by: (a) recruiting beta-arrestin or heterodimerizing with CXCR4; However, the crosstalk between CXCL12/CXCR7/CXCR4 and other signaling mechanisms is more complicated $[42,43]$ (for review, please see Hatterman and Mentlein [35].). The function of CXCR7 is also involved in modulating tumor micro environment; tumor cell migration and apoptosis [42-44].We posit that the inflammatory milieu classically found in leiomyomas induces the CXCL12/ CXCR4/CXCR7 axis leading to a feed-forward cycle of growth within the tumors themselves. Our research is the first study addressing this axis in leiomyomas. Additionally, nonhuman primates develop spontaneous uterine leiomyomas unlike rodent tumor models. This has enormous potential to conduct comparative studies and aims to better understand this disease and eventually develop novel therapeutics targeting the CXCL12/CXCR4/CXCR7 axis for future clinical use. Hence, the novel involvement of CXCR7 as a high affinity binding partner of CXCL12 requires reexamination of the signaling mechanisms [34] involved in inflammatory diseases. Additionally the receptors can switch roles where CXCR7 [36,45] (instead of CXCR4) mediates signal transduction. Further studies on the role of the CXCL12/CXCR4/CXCR7 axis may offer a unique opportunity to treat leiomyomas in a nonsurgical and nonhormonal manner.

\section{Conclusion}

Despite the high prevalence and enormous negative impact of uterine leiomyomas on women's health, their pathogenesis remains largely unidentified. Uterine leiomyomas are associated with several symptoms, including abnormal uterine bleeding, pressure-related symptoms, and recurrent pregnancy loss [1,3-7]. Although the exact etiology is undetermined, the transformation leading to leiomyoma formation is believed to be secondary to a DNA mutation leading to transformation of the myometrial myocyte [46-48]. Research studies show that CXCL12 signaling via receptor CXCR4, is a potent chemokine that attracts stem cells to both normal and abnormal tissues [49]. Since leiomyomas represent an inflammatory milieu, we posit the CXCL12/CXCR4 axis may recruit stem cells to further disease. This suggests targeting the CXCR4/ CXCL12 axis, may translate into a novel therapeutic avenue to treat leiomyomas. Though CXCR4 is the more widely studied receptor for CXCL12, CXCR7 isnow known as an alternate receptor as described above. To date, no data on the role of CXCR7 in leiomyomas has been described.

Although there are rodent xenograft models for studying leiomyomas, this model requires surgical implant into the mouse uterus and does not accurately mimic natural disease. . Leiomyoma research and therapeutic trials would benefit from an animal model with spontaneous uterine leiomyomas similar to humans.
Nonhuman primates involved in aging studies, biomedical research or retired from breeding colonies where tissues have already been harvested when animals oopherectomized or necropsied for other reasons provide a valuable resource. Our ongoing goals follow:

a. Expand the number of human and nonhuman reproductive leiomyomas and compare the expression of CXCL12/CXCR4/ CXCR7 and evaluate tumors with markers for mesenchymal stem markers.

b. Determine the effect of agents that enhance or block CXCL12, CXCR4, or CXCR7 and the effect these experiments have on leiomyoma regression. The overarching goal is to find novel interventions which can reduce or abolish disease as a prerequisite for human trials.

\section{References}

1. Bulun SE, Moravek MB, Yin P, Ono M, Coon JS, et al. (2015) Uterine Leiomyoma Stem Cells: Linking Progesterone to Growth. Semin Reprod Med 33(5): 357-365.

2. Mas A, Stone L, O Connor PM, Yang Q, Kleven D, et al. (2017) Developmental Exposure to Endocrine Disruptors Expands Murine Myometrial Stem Cell Compartment as a Prerequisite to Leiomyoma Tumorigenesis. Stem Cells 35(3): 666-678.

3. Simoens S, Hummelshoj L, D Hooghe T (2007) Endometriosis: cost estimates and methodological perspective. Hum Reprod Update 13(4): 395-404.

4. Donnez J, Donnez O, Orellana R, Binda MM, Dolmans MM (2016) Endometriosis and infertility. Panminerva medica 58(2): 143-150.

5. Giudice LC (2010) Clinical Practice: Endometriosis. The New England journal of medicine 362(25): 2389-2398.

6. Taylor HS, Osteen KG, Bruner-Tran KL, Lockwood CJ, Krikun G, et al. (2011) Novel therapies targeting endometriosis. Reproductive sciences 18(9): 814-823.

7. Sundheimer LW, Alford CE, Taylor RN, DeCherney AH (2000) Endometriosis. In De Groot LJ, Beck-Peccoz P, Chrousos G, et al. eds. Endotext. South Dartmouth (MA).

8. Culley L, Law C, Hudson N, Denny E, Mitchell H, et al. (2013) The social and psychological impact of endometriosis on women's lives: a critical narrative review. Hum Reprod Update 19(6): 625-639.

9. Yang H, Zhou Y, Edelshain B, Schatz F, Lockwood CJ, et al. (2012) FKBP4 is regulated by HOXA10 during decidualization and in endometriosis. Reproduction (Cambridge, England) 143(4): 531-538.

10. Al-Sabbagh M, Lam EW, Brosens JJ (2012) Mechanisms of endometrial progesterone resistance. Molecular and cellular endocrinology 358(2): 208-215.

11. Fazleabas AT (2010) Progesterone resistance in a baboon model of endometriosis. Seminars in reproductive medicine 28(1): 75-80.

12. Cakmak H, Taylor HS (2010) Molecular mechanisms of treatment resistance in endometriosis: the role of progesterone-hox gene interactions. Seminars in reproductive medicine 28(1): 69-74.

13. Aghajanova L, Velarde MC, Giudice LC (2010) Altered gene expression profiling in endometrium: evidence for progesterone resistance. Seminars in reproductive medicine 28(1): 51-58.

14. Bulun SE, Cheng YH, Pavone ME, Xue Q Attar E, et al. (2010) Estrogen receptor-beta, estrogen receptor-alpha, and progesterone resistance in endometriosis. Seminars in reproductive medicine 28(1): 36-43. 
15. Wang C, Mavrogianis PA, Fazleabas AT (2009) Endometriosis is associated with progesterone resistance in the baboon (Papio anubis) oviduct: evidence based on the localization of oviductal glycoprotein 1 (OVGP1). Biol Reprod 80(2): 272-278.

16. Bulun SE, Cheng YH, Yin P, Imir G, Utsunomiya H, et al. (2006) Progesterone resistance in endometriosis: link to failure to metabolize estradiol. Molecular and cellular endocrinology 248(1-2): 94-103.

17. Brown J, Pan A, Hart RJ (2010) Gonadotrophin-releasing hormone analogues for pain associated with endometriosis. The Cochrane database of systematic reviews (12).

18. Sun X, Cheng G, Hao M, Zheng J, Zhou X, et al. (2010) CXCL12 / CXCR4 / CXCR7 chemokine axis and cancer progression. Cancer metastasis reviews 29(4): 709-722.

19. Singh AK, Arya RK, Trivedi AK, Sanyal S, Baral R, et al. (2013) Chemokine receptor trio: CXCR3, CXCR4 and CXCR7 crosstalk via CXCL11 and CXCL12. Cytokine \& growth factor reviews 24(1): 41-49.

20. Werner L, Guzner-Gur H, Dotan I (2013) Involvement of CXCR4/CXCR7 CXCL12 Interactions in Inflammatory bowel disease. Theranostics 3(1): 40-46.

21. Vidakovic M, Grdovic N, Dinic S, Mihailovic M, Uskokovic A, et al. (2015) The Importance of the CXCL12/CXCR4 Axis in Therapeutic Approaches to Diabetes Mellitus Attenuation. Frontiers in immunology 6: 403.

22. Qiao N, Wang L, Wang T, Li H (2016) Inflammatory CXCL12-CXCR4/ CXCR7 axis mediates G-protein signaling pathway to influence the invasion and migration of nasopharyngeal carcinoma cells. Tumour biology: the journal of the International Society for Oncodevelopmental Biology and Medicine 37(6): 8169-8179.

23. Wilkinson M, Walters S, Smith T, Wilkinson A (2008) Reproductive abnormalities in aged female Macaca fascicularis. J Med Primatol 37 Suppl 1: 88-93.

24. Cook AL, Rogers TD, Sowers M (2004) Spontaneous uterine leiomyosarcoma in a rhesus macaque. Contemp Top Lab Anim Sci 43(1): 47-49.

25. Gall AJ, Olds JE, Wunschmann A, Selmic LE, Rasmussen J, et al. (2018) Lesions of the Female Reproductive Tract in Japanese Macaque (Macaca Fuscata) from Two Captive Colonies. J Zoo Wildl Med. 49(1): 79-85.

26. Aristizabal-Arbelaez M, Mejia-Restrepo J, Montoya-Florez M, Grandi F, Pedraza-Ordonez F (2012) Immunohistochemical and morphological features of a small bowel leiomyoma in a black crested macaque (Macaca nigra). BMC Vet Res 8: 97.

27. Dvorska D, Brany D, Dankova Z, Halasova E, Visnovsky J (2017) Molecular and clinical attributes of uterine leiomyomas. Tumour biology: the journal of the International Society for Oncodevelopmental Biology and Medicine 39(6).

28. Akinyemi BO, Adewoye BR, Fakoya TA (2004) Uterine fibroid: a review. Niger J Med 13(4): 318-329.

29. Deshmukh SP, Gonsalves CF, Guglielmo FF, Mitchell DG (2012) Role of MR imaging of uterine leiomyomas before and after embolization. Radiographics 32(6): E251-E281.

30. Prayson RA, Hart WR (1995) Pathologic considerations of uterine smooth muscle tumors. Obstet Gynecol Clin North Am 22(4): 637-657.

31. Don-Salu-Hewage AS, Chan SY, McAndrews KM, MA Chetram, MR Dawson, et al. (2013) Cysteine (C)-x-C receptor 4 undergoes transportin 1-dependent nuclear localization and remains functional at the nucleus of metastatic prostate cancer cells. PLoS One 8(2): e57194.
32. Thelen M, Thelen S (2008) CXCR7, CXCR4 and CXCL12: an eccentric trio? Journal of neuroimmunology 198(1-2): 9-13.

33. Heckmann D, Maier P, Laufs S, Li L, Sleeman JP, et al. (2014) The disparate twins: a comparative study of CXCR4 and CXCR7 in SDF-1alpha-induced gene expression, invasion and chemosensitivity of colon cancer. Clin Cancer Res 20(3): 604-616.

34. Hattermann K, Mentlein R (2013) An infernal trio: the chemokine CXCL12 and its receptors CXCR4 and CXCR7 in tumor biology. Annals of anatomy=Anatomischer Anzeiger: official organ of the Anatomische Gesellschaft 195(2): 103-110.

35. Joo BS, Park MJ, Kim CW, Lee KS, Joo JK (2017) Differential expression of visfatin, leptin, stromal cell derived factor-1alpha, endothelial nitric oxide synthase, and vascular endothelial growth factor in human leiomyomas. Gynecol Endocrinol 33(4): 306-310.

36. Costello CM, McCullagh B, Howell K, Sands M, Belperio JA, et al. (2012) A role for the CXCL12 receptor, CXCR7, in the pathogenesis of human pulmonary vascular disease. Eur Respir J 39(6): 1415-1424.

37. Li XJ, Liu P, Tian WW, Li ZF, Liu BG, et al. (2017) Mechanisms of CXCR7 induction in malignant melanoma development. Oncol Lett 14(4): 41064114

38. Yao X, Zhou L, Han S, Chen Y (2011) High expression of CXCR4 and CXCR7 predicts poor survival in gallbladder cancer. J Int Med Res 39(4): 1253-1264.

39. Chang K, Li GX, Kong YY, shen XX, Qu YY, et al. (2017) Chemokine Receptors CXCR4 and CXCR7 are Associated with Tumor Aggressiveness and Prognosis in Extramammary Paget Disease. J Cancer 8(13): 24712477.

40. Dai X, Tan Y, Cai S, Xiong X, Wang L, et al. (2011) The role of CXCR7 on the adhesion, proliferation and angiogenesis of endothelial progenitor cells. Journal of cellular and molecular medicine 15(6): 1299-1309.

41. D Alterio C, Consales C, Polimeno M, Franco R, Cindolo L, et al. (2010) Concomitant CXCR4 and CXCR7 expression predicts poor prognosis in renal cancer. Curr Cancer Drug Targets 10(7): 772-781.

42. Xu D, Li R, Wu J, Jiang L, Zhong HA (2016) Drug Design Targeting the CXCR4/CXCR7/CXCL12 Pathway. Curr Top Med Chem 16(13): 14411451.

43. Sanchez-Martin L, Sanchez-Mateos P, Cabanas C (2013) CXCR7 impact on CXCL12 biology and disease. Trends in molecular medicine 19(1): $12-22$

44. Wang L, Wang Z, Yang B, Yang Q, Wang L, et al. (2009) CXCR4 nuclear localization follows binding of its ligand SDF-1 and occurs in metastatic but not primary renal cell carcinoma. Oncol Rep 22(6): 1333-1339.

45. Tripathi V, Kumar R, Dinda AK, Kaur J, Luthra K (2014) CXCL12-CXCR7 signaling activates ERK and Akt pathways in human choriocarcinoma cells. Cell Commun Adhes 21(4): 221-228.

46. Panagopoulos I, Gorunova L, Brunetti M, Agostini A, Andersen HK, et al. (2017) Genetic heterogeneity in leiomyomas of deep soft tissue. Oncotarget 8(30): 48769-48781.

47. Ordulu Z (2016) Fibroids: Genotype and Phenotype. Clin Obstet Gynecol 59(1): 25-29.

48. Yang Q Diamond MP,Al-Hendy A (2016) Early Life Adverse Environmental Exposures Increase the Risk of Uterine Fibroid Development: Role of Epigenetic Regulation. Front Pharmacol 7: 40.

49. Hocking AM (2015) The Role of Chemokines in Mesenchymal Stem Cell Homing to Wounds. Adv WoundCare (New Rochelle) 4(11): 623-630. 


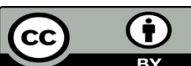

This work is licensed under Creative Commons Attribution 4.0 License

Submission Link:

Submit Article

DOI: 10.32474/OAJRSD.2018.01.000108

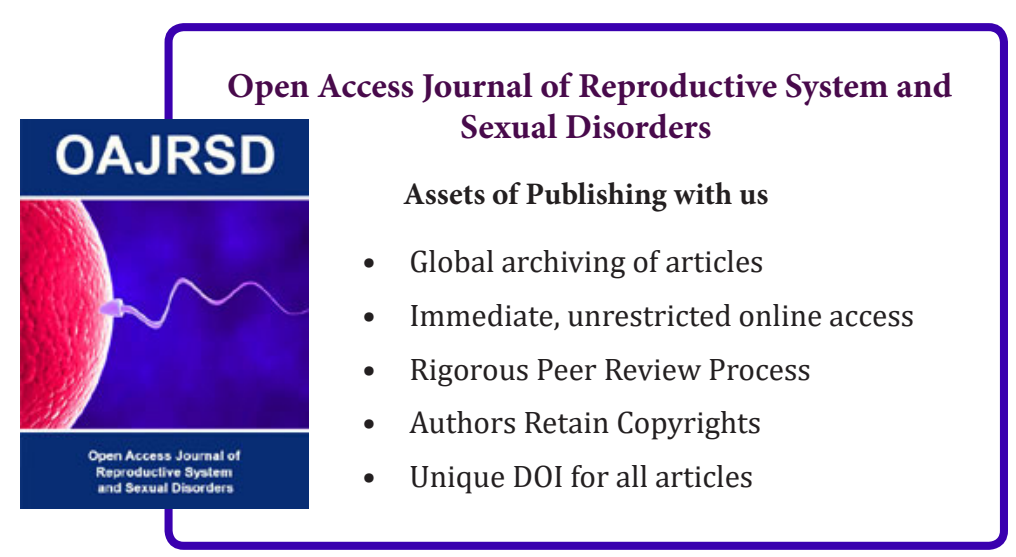

